\title{
Assembly of lipase and P450 fatty acid decarboxylase to constitute a novel biosynthetic pathway for production of 1-alkenes from renewable triacylglycerols and oils
}

Jinyong Yan ${ }^{1 *}$, Yi Liu ${ }^{1,2}$, Cong Wang ${ }^{1}$, Bingnan $\operatorname{Han}^{3}$ and Shengying $\mathrm{Li}^{\mathrm{i}^{*}}$

\begin{abstract}
Background: Biogenic hydrocarbons (biohydrocarbons) are broadly accepted to be the ideal 'drop-in' biofuel alternative to petroleum-based fuels due to their highly similar chemical composition and physical characteristics. The biological production of aliphatic hydrocarbons is largely dependent on engineering of the complicated enzymatic network surrounding fatty acid biosynthesis.

Result: In this work, we developed a novel system for bioproduction of terminal fatty alkenes (1-alkenes) from renewable and low-cost triacylglycerols (TAGs) based on the lipase hydrolysis coupled to the P450 catalyzed decarboxylation. This artificial biosynthetic pathway was constituted using both cell-free systems including purified enzymes or cell-free extracts, and cell-based systems including mixed resting cells or growing cells. The issues of high cost of fatty acid feedstock and complicated biosynthesis network were addressed by replacement of the de novo biosynthesized fatty acids with the fed cheap TAGs. This recombinant tandem enzymatic pathway consisting of the Thermomyces lanuginosus lipase (TII) and the P450 fatty acid decarboxylase Ole $T_{\text {JE }}$ resulted in the production of 1-alkenes from purified TAGs or natural oils with 6.7 to $46.0 \%$ yields.

Conclusion: Since this novel hydrocarbon-producing pathway only requires two catalytically efficient enzymatic steps, it may hold great potential for industrial application by fulfilling the large-scale and cost-effective conversion of renewable TAGs into biohydrocarbons. This work highlights the power of designing and implementing an artificial pathway for production of advanced biofuels.
\end{abstract}

Keywords: Lipase, P450 fatty acid decarboxylase, Alkenes, Cell-free catalysis, Whole cell catalysis

\section{Introduction}

Shortage of petroleum-based fuels and increasing environmental concerns have led to great efforts to develop sustainable and clean biofuels from renewable feedstocks. Bioethanol manufactured by fermentation of sugars and biodiesel produced via transesterification of vegetable oils, animal fat, or waste oils are regarded as the two major first-generation biofuels, which are dominating the current global market of biofuels [1]. However, some

\footnotetext{
* Correspondence: yjiny@126.com; lishengying@qibebt.ac.cn

'Key Laboratory of Biofuels, and Shandong Provincial Key Laboratory of

Energy Genetics, Qingdao Institute of Bioenergy and Bioprocess Technology, Chinese Academy of Sciences, No. 189 Songling Road, 266101 Qingdao,

Shandong, China

Full list of author information is available at the end of the article
}

undesirable properties such as high miscibility with water and low energy density (for bioethanol), incompatibility with current engine systems, and problems associated with storage and distribution have limited their further popularization [2]. Therefore, advanced biofuels with better fuel properties have been receiving ever-increasing attention, among which biohydrocarbons, especially the medium- to long-chain fatty alkanes/ alkenes, are considered the ideal alternatives to petroleum-based transportation fuels due to their highly similar chemical composition and physical characteristics. Thus, biohydrocarbons hold the potential to be developed into 'drop-in' biofuels compatible with the existing distribution infrastructures used for gasoline, 
diesel, and jet fuels in terms of their fatty acyl chain length [3].

At present, chemical hydrotreatment of acylglycerides, fatty acids, or fatty acyl esters, and de novo microbial biosynthesis are the two major strategies for production of biohydrocarbons. The former strategy requires expensive metal catalysts (for example, $\mathrm{Pd}$ and $\mathrm{Pt}$ ), high temperature $\left(250\right.$ to $450^{\circ} \mathrm{C}$ ), and high pressure (20 to 70 bar), hence being energy intensive and environmentally unfriendly [4]. In comparison, the latter bioproduction strategy highlighted by a limited number of metabolically engineered systems is undoubtedly 'greener' [5-10]. However, all these biosynthetic systems suffer greatly from low yield of hydrocarbons, preventing them from industrialization.

For instance, the first engineered alkane biosynthetic pathway consisting of the acyl-acyl carrier protein (ACP) reductase Orf1594 from Synechococcus elongates PCC7942 and the aldehyde decarbonylase from Nostoc punctiforme PCC73102 was constructed in Escherichia coli and gave rise to a total alkane titer of approximately $300 \mathrm{mg} / \mathrm{l}$ [5]. Later, two similar hybrid biosynthetic routes by coupling a carboxylic acid reductase or a fatty acid reductase complex to the same fatty aldehyde decarbonylase were also reconstituted, however, leading to lower alkane yields [6,7]. Recently, based on the in vivo activity of the long-chain fatty acid P450 decarboxylase $\mathrm{OleT}_{\mathrm{JE}}$, our laboratory engineered a series of E. coli strains capable of de novo biosynthesis of 1-alkenes from glucose with the highest total alkene titer of $96.7 \mathrm{mg} / \mathrm{l}$ [10].

All these engineered pathways were constructed through diverting the fatty acid biosynthetic pathway toward hydrocarbon synthesis via different mechanisms of deoxygenation (Figure 1A). Since fatty acid biosynthesis plays a central role in energy metabolism of all living organisms and involves multiple interplaying enzymatic steps [11-17], its regulation is highly complicated and hence difficult to be manipulated. Metabolic engineering efforts aimed at overproduction of fatty acids or their derivatives (for example, fatty acyl-ACPs or fatty acyl CoAs) for high production of hydrocarbons via genetically reprogramming the fatty acid biosynthetic system turn out to be intrinsically difficult and technically challenging.

To overcome the limit of de novo biosynthesis of hydrocarbons, in this study, we propose a novel strategy for long-chain fatty alkene biosynthesis through coupling the glyceride hydrolytic activity of the Thermomyces lanuginosus lipase (Tll) to the fatty acid decarboxylation activity of the P450 enzyme OleT $\mathrm{T}_{\mathrm{IE}}$ (Figure 1B). Tll has been well characterized as a biocatalyst with remarkable capacity of hydrolyzing triacylglycerols (TAGs) to free fatty acids (FFAs) involved in oil modifications [18]. $\mathrm{OleT}_{\mathrm{JE}}$ was recently discovered as a novel P450 enzyme with the unique ability to decarboxylate long-chain FFAs, forming terminal alkenes (1-alkenes) [19]. It is evident that FFAs should be able to couple these two naturally unrelated reactions. Thus, we selected these two enzymes to construct an artificial two-step biosynthetic pathway for biological production of 1-alkenes.

In the pilot biosynthetic system based on purified enzymes, the exogenous feedstock including pure TAGs and natural oils were first efficiently hydrolyzed by Tll, and the released free fatty acids (FFAs) were decarboxylated by $\mathrm{OleT}_{\mathrm{JE}}$ resulting in 1-alkenes (Graphical abstract in Additional file 1). Upon the proof-of-concept by using pure enzymes, the cell-based systems including the mixed resting cells that express Tll and $\mathrm{OleT}_{\mathrm{IE}}$, respectively, and the resting cells that co-express $\mathrm{Tll}$ and $\mathrm{OleT}_{\mathrm{JE}}$ were also evaluated. Moreover, the in situ catalytic system that is capable of mediating the biotransformation of TAGs $\rightarrow$ FFAs $\rightarrow 1$-alkenes during cell growth was further customized from the perspective of cost-effective production of alkenes at industrial scale. This innovative tandem biotransformation process for producing olefin hydrocarbons from TAGs broadens the application of lipase in biofuel synthesis, which has long been only focused on biodiesel production [20-22]. The simple two-step (hydrolysis followed by decarboxylation) enzymatic pathway appears to be efficient and more manageable compared to the complex fatty acid biosynthetic network. Since TAGs are widely present in plant oils, animal fats, and oleaginous microbial cells [23], the feedstock for future large-scale application of this new biohydrocarbonproducing strategy should be abundant and cost-effective.

\section{Results and discussion}

Conversion of TAGs $\rightarrow$ FFAs $\rightarrow$ 1-alkenes catalyzed by purified TII and OleT $_{\text {JE }}$

The two enzymes $\operatorname{OleT}_{\mathrm{JE}}$ and Tll were functionally expressed in E. coli and purified to homogeneity (Figure $\mathrm{S} 1$ in Additional file 2). In the lipase-catalyzed hydrolysis of pure TAGs $(0.5 \mathrm{mM})$ that only have C12, C14, and C16 fatty acyl chains (that is, trilaurin, trimyristin, and tripalmitin), Tll released $0.80 \mathrm{mM}$ lauric acid (C12), $0.78 \mathrm{mM}$ myristic acid (C14), and $1.15 \mathrm{mM}$ palmitic acid (C16), corresponding to $53.3 \pm 1.3,52.0 \pm 1.1$, and $76.7 \pm 1.5 \%$ yield (Figure $2 \mathrm{~A}$ ), respectively.

To test whether the fatty acid decarboxylation activity of $\mathrm{OleT}_{\mathrm{JE}}$ can be coupled to the lipase-catalyzed hydrolysis in one pot, $2 \mu \mathrm{M} \mathrm{OleT} \mathrm{TE}_{\mathrm{JE}}$ and $1 \mathrm{mM} \mathrm{H}_{2} \mathrm{O}_{2}$ as cofactor were added into the TAG hydrolytic reactions. As expected, $0.35 \mathrm{mM}$ 1-undecene (C11), $0.49 \mathrm{mM}$ 1-tridecene (C13), and $0.40 \mathrm{mM}$ 1-pentadecene $(\mathrm{C} 15)$ were produced (Figure 2B), corresponding to $23.3 \pm 0.9,32.7 \pm 1.4$, and $26.7 \pm 0.9 \%$ of the theoretically maximal conversion from TAG to corresponding 1-alkene. When only taking the second decarboxylation step into account, as 


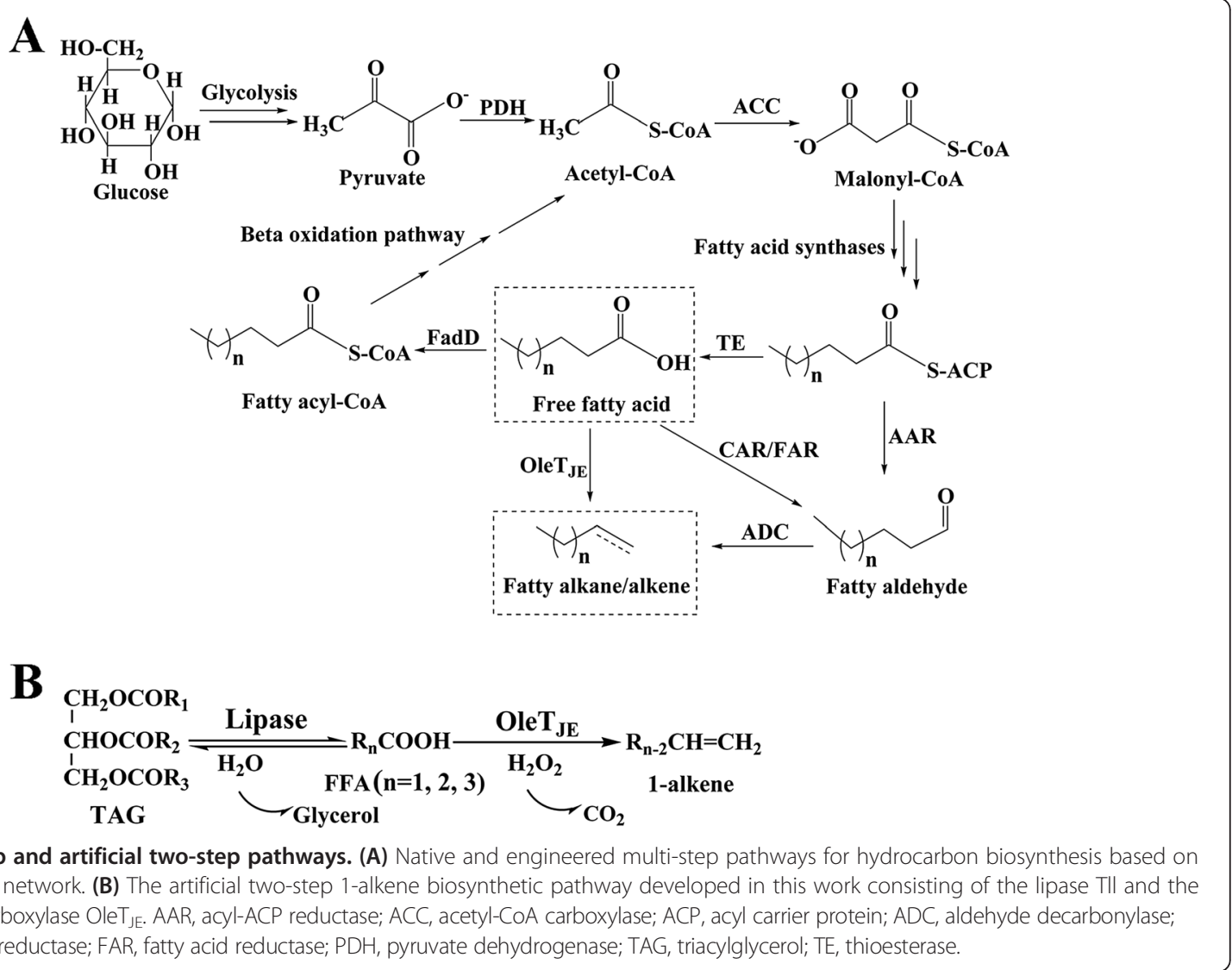

shown in Figure 2B, the conversions of the $\mathrm{C} 12, \mathrm{C} 14$, and C16 FFA to corresponding 1-alkene were $39.8 \pm 1.5$, $58.3 \pm 1.3$, and $33.3 \pm 1.1 \%$, respectively. These results evidently demonstrate the compatibility between the reactions mediated by $\mathrm{Tll}$ and $\mathrm{OleT}_{\mathrm{JE}}$. Among the tested TAG hydrolysis reactions, Tll displayed the highest activity toward tripalmitin (C16 TAG), which indicates the fatty acyl chain length preference of Tll being $\mathrm{C} 16$ over $\mathrm{C} 14$ and $\mathrm{C} 12$. By contrast, in the tandem hydrolysis-decarboxylation process, the alkene product with the greatest yield turned out to be 1-tridecene (Figure 2B), decarboxylated from the C14 FFA-myristic acid. In the one-pot catalytic relay, the consumption of fatty acids by $\mathrm{OleT}_{\mathrm{JE}}$ (forming alkenes) was expected to pull the equilibrium of TAGs $\leftrightarrow$ fatty acids toward fatty acids (Figure 1B). Consistently, the total FFA yield of the tested dual enzymatic systems calculated by summing up the reacted FFA (equals to the produced alkene in mole) and the remaining FFA (Figure 2B) was slightly improved compared to that of the single lipase hydrolytic system (Figure 2A).

In a mixed biocatalytic system of dual enzymes, the ratio between the two enzymes is often a key factor for the overall conversion ratio. Thus, we elected to use trimyristin (the TAG with three C14 fatty acyl chains), which gave the highest 1-alkene yield at a 1:1 enzyme ratio (Figure 2B), as substrate to optimize the proportion of lipase to decarboxylase. As shown in Figure 2C, the C13 alkene yield was improved to $0.69 \mathrm{mM}$ (corresponding to $46.0 \pm 1.8 \%$ conversion of TAG to alkene) at a molar ratio of 1:2 (Tll:OleT $\mathrm{JE}_{\mathrm{JE}}$ ). It was reported that the extensive metabolic engineering efforts aimed at overproduction of FFAs in E. coli have so far achieved less than 30\% of the maximum theoretical yield via de novo biosynthesis from the starting carbon source glucose [16]. The reported yields of FFA downstream products such as hydrocarbons were even lower [5-7,10]. Thus, the $46.0 \%$ overall conversion from TAG to 1-alkene achieved by the cell-free system of tandem lipase-OleT $\mathrm{JE}_{\mathrm{J}}$ in this work appears significant. The in vitro manipulation via enzyme ratio optimization held another significant advantage over the less accurate in vivo metabolic engineering, which likely requires a delicate coordination of the complicated regulation networks of transcription, translation, and metabolite fluxes.

Subsequently, three natural oils other than pure TAGs including olive oil, soybean oil, and microalgae oil were investigated as substrates for the novel Tll-Ole $\mathrm{T}_{\mathrm{JE}}$ catalytic relay system. The Tll-catalyzed hydrolysis gave rise to $1.21,1.12$, and $1.37 \mathrm{mM}$ total FFAs from olive oil, 

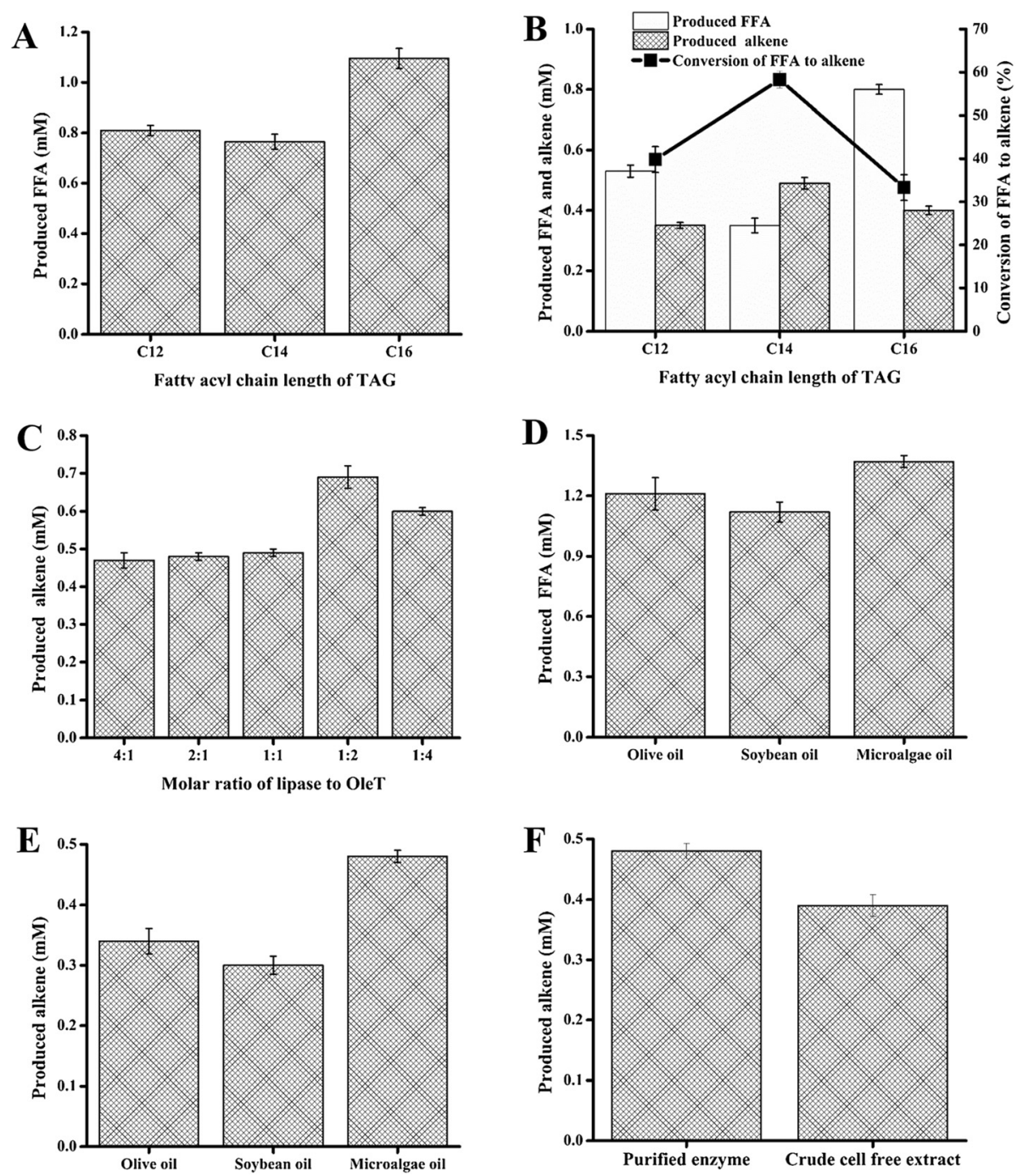

Figure 2 Production of FFAs and 1-alkenes and optimization of the enzyme ration between TIl and OleT JE. (A) Production of FFAs from hydrolysis of different TAGs by purified TII. (B) Production of FFAs and 1-alkenes from TAGs catalyzed by the coupled TII-OleT JE. (C) Optimization of the enzyme ratio between TII and OleT JE for improvement of the overall alkene yield from the TAG with C14 fatty acyl chain length. (D) Production of FFAs from hydrolysis of different natural oils by purified TII. (E) Production of 1-alkenes from natural oils catalyzed by the coupled TII-OleT JE. (F) Production of 1-alkenes from microalgae oil mediated by purified enzymes and cell-free extracts. FFA, free fatty acid; TAG, triacylglycerol.

soybean oil, and microalgae oil (Figure 2D), corresponding to $80.7 \pm 2.3,74.7 \pm 2.1$, and $91.3 \% \pm 3.3$ conversion of TAGs to FFAs, respectively. The profiles of produced FFAs are described in Table S1 in Additional file 3. In the coupled hydrolysis-decarboxylation reactions, 0.34 , 0.30 , and $0.48 \mathrm{mM}$ total alkenes including 1-tridecene (for microalgae oil only), 1-pentadecene, and 1-heptadecene, corresponding to $21.3 \pm 0.7,20.0 \pm 0.9$, and $32.0 \pm 1.0$ overall conversion from TAGs to alkenes (Figure 2E; Table S1 in Additional file 3), were produced from olive oil, soybean oil, and microalgae oil, respectively. Interestingly, no higher alkenes were observed in this coupled reaction system, although FFAs of C20:5 and C22:6 were released from microalgae oil. This indicates the relatively lower substrate flexibility of $\mathrm{OleT}_{\mathrm{JE}}$ than that of Tll, which is consistent with the reported fatty acid chain length selectivity of saturated C12 to C20 [10,19]. Comparatively, microalgae oil appeared to be a more suitable feedstock for 1-alkene production via tandem biocatalysis. Since microalgae oils could be an excellent feedstock for this biosynthetic pathway to produce advanced hydrocarbon biofuels, all following experiments used this feedstock.

To reduce the biocatalyst cost by omitting the enzyme purification step, the crude E. coli cell-free extracts of Tll and $\operatorname{OleT}_{\mathrm{JE}}$ were applied for the production of 1-alkene using microalgae oil as feedstock. As a result, $0.39 \mathrm{mM}$ 
1-alkenes were generated, comparable to the $0.48 \mathrm{mM}$ yield when using purified enzymes (Figure 2F).

\section{Production of 1-alkenes mediated by mixed resting E. coli recombinant cells}

Biotransformation mediated by whole cell catalysts represents a promising strategy for industrial manufacture because it skips the costly enzyme purification and immobilization steps. To reduce the cost on biocatalysts, the two E. coli whole cell catalysts of Tll and $\mathrm{OleT}_{\mathrm{JE}}$ were mixed and evaluated. Importantly, in the mixed cell system, the enzyme ratio can be easily controlled by adjusting cell dosage. However, multiple events of substrate/product transfer across cell membrane must be considered.

For the TAG hydrolytic reactions mediated by overexpressed cytoplasmic Tll in E. coli/pRSFDuet-tll whole cells, freeze-dried cells hydrolyzed $70 \%$ microalgae oil to form 1.05 mM FFAs (Figure 3A), which are more catalytically efficient than non-treated cells $(0.9 \mathrm{mM}, 60 \pm 2.3 \%)$. This indicates freeze-drying is a simple but effective way to improve substrate accessibility and hence catalytic activity.

To further improve mass transfer of TAG substrates, Tll was periplasmically overexpressed in E. coli/pET-22 (b)-tll in order to hydrolyze TAGs more efficiently. As expected, a higher yield of $80 \%$ (1.2 mM FFAs produced) was achieved (Figure 3A). For periplasmic overexpression of Tll in E. coli, fusion of the Tll lipase to the pelB leader sequence directs the lipase to E. coli periplasm, where the leader peptide is removed by a signal peptidase [24]. Considering that the two types of cells used for biotransformations contained the same total activities (normalized in terms of cell-free extract), we reason that the enhancement of FFA production catalyzed by periplasmic Tll overexpression cells was likely attributed to improved substrate accessibility by localization of enzymes in periplasm. The freeze-dried E. coli/pET-22(b)-tll barely increased the degree of hydrolysis (Figure 3A).

In the case of Tll-Ole $\mathrm{T}_{\mathrm{IE}}$ coupled reaction in the mixed cell system, E. coli/pET-22(b)-tll (for periplasmic expression of Tll) plus E. coli/pACYCDuet-ole $T_{J E}$ (for cytoplasmic expression of $\left.\mathrm{OleT}_{\mathrm{JE}}\right)$ gave higher alkene yield $(0.21 \mathrm{mM}, 14 \pm 0.4 \%)$ than that $(0.10 \mathrm{mM}, 7 \pm 0.3 \%)$ of $E$. coli/pRSFDuet-tll (for cytoplasmic expression of Tll) plus E. coli/pACYCDuet-ole $T_{J E}$ (for cytoplasmic expression of $\mathrm{OleT}_{\mathrm{JE}}$ ) (Figure $3 \mathrm{~B}$ ). Also shown in Figure 3B, the mixed freeze-dried cells displayed higher conversion than the non-treated counterparts for the group with cytoplasmically overexpressed Tll (E. coli/pRSFDuet-tll $+E$.
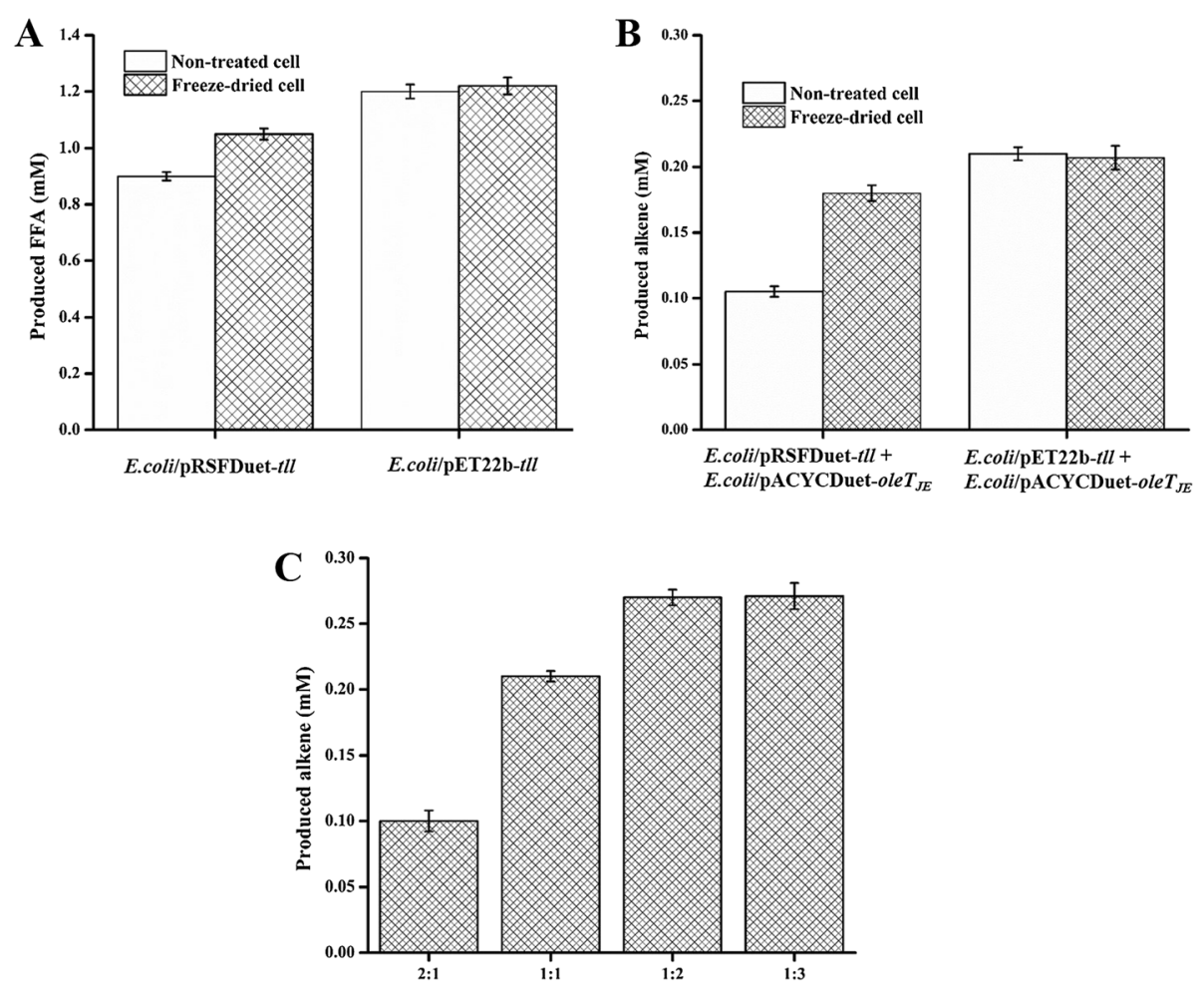

Figure 3 Production of FFAs and 1-alkenes from microalgae oil and optimization of enzyme ratio. (A) Production of FFAs from microalgae oil by resting E. coli cells with cytoplasmic and periplasmic overexpression of TIl. (B) Production of 1-alkenes from microalgae oil catalyzed by mixed resting E. coli cells. (C) Optimization of the enzyme ratio between TII and OleT JE in the form of freeze-dried whole cell catalysts for improvement of the overall alkene yield from microalgae oil. 
coli/pACYCDuet-ole $T_{J E}$ ), but there was only a slight improvement for the group with periplasmic overexpression of Tll (E. coli/pET-22(b)-tll $+E$. coli $/ \mathrm{pACYC}-$ Duet-ole $\left.T_{J E}\right)$.

Further optimization of the enzyme ratio in the form of freeze-dried cells (E. coli/pET-22(b)-tll $+E$. colil pACYCDuet-ole $T_{J E}$ ) enhanced the yield to $18 \pm 0.8 \%$ $(0.27 \mathrm{mM})$ at a 1:2 molar ratio (Tll:Ole $\left.\mathrm{T}_{\mathrm{JE}}\right)$ (Figure $\left.3 \mathrm{C}\right)$. Although the ratio between the two enzymes can be adjusted in the mixed cell system, the catalytic efficiency of the whole system apparently would suffer from complicated transportation of intermediate FFAs from one type of cells to another. This is likely the major reason for the low conversions from TAGs to 1-alkenes. Therefore, engineering of an $E$. coli strain with co-expression of the two enzymes seems necessary.

\section{Biosynthesis of 1-alkenes with a whole cell catalyst co- expressing $\mathrm{TII}$ and $\mathrm{OleT}_{\mathrm{JE}}$}

The approach of co-expressing Tll (for oil hydrolysis) and $\mathrm{OleT}_{\mathrm{JE}}$ (for alkene formation) in the same E. coli host could potentially overcome complex cross-membrane transport of intermediate FFAs between different types of cells, thereby gaining more efficient substrate channeling. Thus, we constructed two types of co-expression strains including $E$. coli/pRSFDuet-tll + pACYCDuet-ole $T_{J E}$ and E. coli/pET-22(b)-tll + pACYCDuet-ole $T_{J E}$ and evaluated their alkene-producing abilities. The non-treated E. coli cells with cytoplasmic co-expression of Tll and $\mathrm{OleT}_{\mathrm{JE}}$ (E. coli/pRSFDuet-tll + pACYCDuet-ole $T_{J E}$ ) converted $11 \pm 0.7 \%$ microalgae oil into 1-alkenes $(0.16 \mathrm{mM})$, whereas the freeze-dried cells gave a higher yield of $17 \pm$ $1.1 \%(0.26 \mathrm{mM})$ (Figure 4A), both of which are greater than that of corresponding mixed cell systems. This improvement can be explained by better channeling of intermediates between adjacent enzyme active sites because of co-localization of two enzymes inside a single cell.
A similar profile was observed for the periplasmic co-overexpression cell system (E. coli/pET-22(b)-tll + pACYCDuet-ole $\left.T_{J E}\right)$. Specifically, the strain of $E$. coli/ pET-22(b)-tll + pACYCDuet-ole $T_{J E}$ demonstrated improved hydrocarbon yield $(0.35 \mathrm{mM}, 24 \pm 1.3 \%)$ compared to the corresponding cytoplasmic overexpression cell system (E. coli/pRSFDuet-tll + pACYCDuet-ole $\left.T_{J E}\right)$. Again, this result indicates the importance of the contact between TAGs and Tll for the whole alkene-producing pathway. The freeze-dried periplasmic overexpression cells $\left(E\right.$. coli/pET-22(b)-tll + pACYCDuet-ole $\left.T_{J E}\right)$ did not enhance the conversion (0.36 mM, 24 $\pm 1.4 \%$ ) (Figure 4A), indicating that freeze-drying treatment cannot further improve the cell permeabilization that has already been optimized by periplasmic expression of Tll. We infer that periplasmic expression of Tll probably leads to better permeability than the corresponding cytoplasmic expression; thus, the freeze-drying treatment showed a positive permeabilization effect on the cytoplasmic co-expression cell type, but not on the periplasmic co-expression type.

Measurement of the activity of the two enzymes contained in co-expression cells showed molar ratios of 1:1.7 and 1:1.9 (Tll:OleT ${ }_{\mathrm{JE}}$ ), respectively, close to the optimal ratio of 1:2. More significantly, the periplasmic co-expression cells retained more than $70 \pm 3.2 \%$ alkene-producing activity after recycling in three successive batches, displaying great potential for industrial application.

\section{Biosynthesis of 1-alkenes by co-expressed TII and OleT JE in situ during cell growth}

Integration of enzyme generation and enzymatic transformation into a single process would be significantly more energy efficient and of industrial simplification by making better use of fermentation energy and skipping enzyme purification. We previously developed an integrated bioprocess for in situ biodiesel production that occurs simultaneously with lipase generation in a Pichia
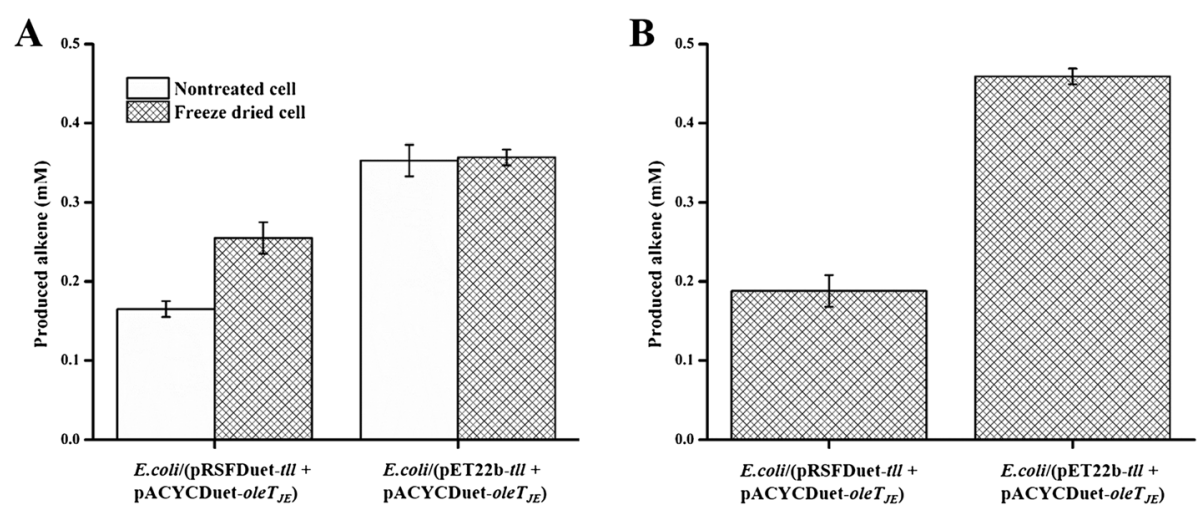

Figure 4 Production of 1-alkenes from microalgae oil. (A) Production of 1-alkenes from microalgae oil catalyzed by E. coli resting cells with co-expression of Tll and OleTJE. (B) Production of 1-alkenes from microalgae oil catalyzed by growing in situ E. coli cell catalysts. 
pastoris yeast system [25]. Inspired by this strategy, in the present study, in parallel with combining hydrolysis of TAGs and decarboxylation of FFAs into a single $E$. coli host, we tested the strategy of developing E. coli in situ catalytic system for biosynthesis of 1-alkenes from TAGs. This in situ system integrated the process of enzyme production with the enzyme-catalyzed tandem biotransformations into a single process in one pot, which could significantly save energy consumption and simplify operational procedures.

Based on the tandem hydrolysis-decarboxylation in situ catalyzed by Tll and $\mathrm{OleT}_{\mathrm{JE}}$ during cell growth, the two types of Tll-OleT $\mathrm{T}_{\mathrm{JE}}$ co-expression $E$. coli cells $(E$. coli/ pRSFDuet-tll + pACYCDuet-ole $T_{J E}$ and $E$. coli/pET-22(b)$t l l+$ pACYCDuet-ole $\left.T_{J E}\right)$ gave $0.19 \mathrm{mM}(12 \pm 0.6 \%)$ and $0.46 \mathrm{mM}(31 \pm 1.1 \%)$ alkene yield from the fed microalgae oils, respectively (Figure 4B). Interestingly, periplasmic overexpression cells (E. coli/pET-22(b)-tll + pACYCDuetole $_{J E}$ ) even secreted some lipases into the extracellular space (that is, culture medium) during the in situ process, which may significantly facilitate the hydrolysisdecarboxylation coupled reactions, as reflected by enhanced alkene production. Thus, the in situ catalytic system based on periplasmic overexpression could take advantage of both cell-free enzymes and whole cell catalysts. The extracellular and intracellular enzymes of growing $E$. coli cells were likely to be simultaneously utilized to produce alkenes in situ and in one pot. Moreover, we further evaluated this in situ system by recovering the whole cells and applied them for a new batch reaction as resting whole cell catalysts, which displayed $61.0 \pm 2.2$ and $73.0 \pm 2.9 \%$ relative yield for cytoplasmic overexpression cells $(E$. coli/pRSFDuet- $t l l+$ pACYCDuet-ole $T_{J E}$ ) and periplasmic overexpression cells (E. coli/pET-22(b)-tll + pACYCDuet-ole $T_{J E}$ ), respectively.

Notably, higher concentrations of the substrate oil (1 to $5 \mathrm{mM}$ ) or $\mathrm{H}_{2} \mathrm{O}_{2}$ (1 to $15 \mathrm{mM}$ ) were also tested; however, the alkene yields did not increase (data not shown). It is possible that the low activity or low tolerance of $\mathrm{OleT}_{\mathrm{JE}}$ toward the oil-FFA mixture may limit higher alkene yield. Thus, based on the crystal structure of $\mathrm{OleT}_{\mathrm{JE}}$ recently reported by Belcher et al. [26], the protein engineering of this enzyme to improve its activity or tolerance in the context of two-step reactions could be helpful for developing a more efficient converter microorganism.

Compared to the in vivo construction and regulation of metabolic pathways, the in vitro strategy of cell-free enzyme-based and whole cell-based systems represents an important opportunity for bioproduction of hydrocarbons. These in vitro systems could be readily controlled through preparation of enzyme or whole cell cocktails by simple mixing procedures or accurate modification of reaction conditions $[27,28]$. These multiple cell-free enzyme systems (purified enzymes, cell-free extracts) and cell-based systems (mixed resting cells, whole cells with co-expressed enzymes, growing in-situ cell catalysts) provide more flexible choices for specific purpose and situation (Figure 5).

\section{Conclusion}

A novel biosynthetic pathway for production of biohydrocarbon 1-alkenes from renewable oils was assembled, which consists of the lipase Tll to release FFAs from TAGs and the P450 fatty acid decarboxylase $\mathrm{OleT}_{\mathrm{JE}}$ responsible for decarboxylation of FFAs to generate 1alkenes. The biotransformation of TAGs $\rightarrow$ FFAs $\rightarrow 1$-alkenes was achieved using a variety of biocatalytic systems, including purified enzymes, cell-free extracts, resting whole cells, and growing in situ cell catalysts. These onepot tandem biocatalyzes only required two sequential enzymatic steps, but allowed the efficient production of 1alkenes from TAGs with 6.7 to $46.0 \%$ conversion ratios from $0.5 \mathrm{mM}$ renewable feedstock including pure TAGs and diverse natural oils. Especially, the biosynthesis of 1alkenes based on resting whole cells and growing in situ cell catalysts exhibits potential for industrial application.

Finally, it is worth noting that this novel artificial biosynthetic route could be adapted for other microorganisms, especially for those genetically tractable oleaginous microorganisms if considering the abundant endogenous TAGs as potential cellular substrates for the Tll-OleT $\mathrm{JE}_{\mathrm{JE}}$ coupled system.

\section{Materials and methods}

\section{Plasmids, strains, and reagents}

The plasmids pACYCDuet-1, pRSFDuet-1, and pET-22 (b) were obtained from Novagen (Darmstadt, Germany). Recombinant plasmid pET-28(b)-ole $T_{J E}$ for expression of the $\mathrm{P} 450$ fatty acid decarboxylase $\mathrm{OleT}_{\mathrm{JE}}$ was constructed in our laboratory previously [10]. E. coli DH5 $\alpha$ and BL21 (DE3) strains were preserved in our laboratory. Various authentic standards of TAGs (C12, trilaurin, C14, trimyristin, and C16, tripalmitin), fatty acids (lauric acid, myristic acid, palmitic acid, stearic acid, and heptadecanoic acid), and fatty 1 -alkenes (1-undecene, 1-tridecene, 1-pentadecene, and 1-heptadecene) were purchased from TCI (Shanghai, China). Kanamycin, chloramphenicol, thiamine, and isopropyl $\beta$-D-1-thiogalactopyranoside (IPTG) were products of Solarbio Science \& Technology Co., Ltd (Beijing, China). All used restricted enzymes were supplied by Thermo Scientific (Shanghai, China). PrimeSTAR GXL DNA polymerase and dNTPs were from Takara Bio Inc. (Otsu, Japan). DNA manipulation kits were bought from Omega Bio-Tek (Norcross, GA, USA) and Promega (Madison, WI, USA). Ni-NTA resin was from Qiagen (Venlo, Netherlands). PD-10 desalting columns were products of GE Healthcare (Pewaukee, WI, USA). Ultra centrifugal 

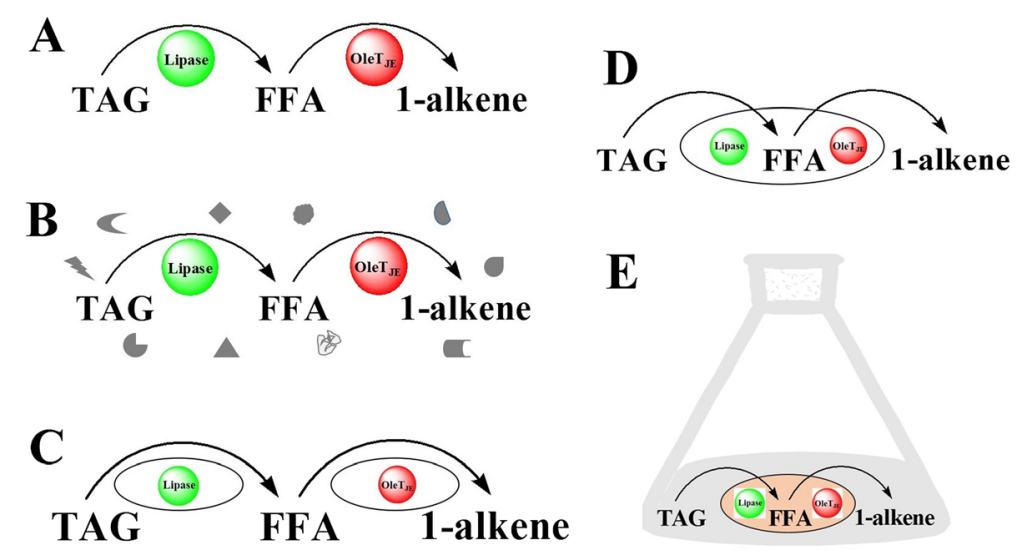

Figure 5 Catalytic systems for the conversion of triacylglycerol to 1-alkene. Various lipase-OleT JE coupling catalytic systems for the conversion of triacylglycerol to 1-alkene including (A) purified enzymes, (B) cell-free extract, (C) mixed resting cells, (D) co-expression cells, and (E) in situ growing cells. FFA, free fatty acid; TAG, triacylglycerol.

filters were purchased from Millipore (Billerica, MA, USA). Bradford Protein Assay kit was bought from Beyotime Institute of Biotechnology (Jiangsu, China). Other routine reagents were commercially available products of analytical grade. The tll gene was synthesized by GenScript (Piscataway, NJ, USA). Oligo primers were synthesized by Sangon Biotech (Shanghai, China). Soybean oil and olive oil were purchased from local market. Microalgae oil was kindly donated by Professor Tianzhong Liu at the Qingdao Institute of Bioenergy and Bioprocess Technology, Chinese Academy of Sciences.

TB medium was composed of $1.2 \%$ tryptone, $2.4 \%$ yeast extract, $0.5 \%$ glycerol, $0.23 \% \mathrm{KH}_{2} \mathrm{PO}_{4}$, and $1.25 \%$ $\mathrm{K}_{2} \mathrm{HPO}_{4}$. One millimolar of thiamine was supplemented for the expression of $\mathrm{OleT}_{\mathrm{JE}}$. For maintaining corresponding plasmids, $25 \mu \mathrm{g} / \mathrm{ml}$ chloramphenicol or $50 \mu \mathrm{g} / \mathrm{ml}$ kanamycin was added.

The lysis buffer ( $\mathrm{pH}$ 8.0) was composed of $\mathrm{NaH}_{2} \mathrm{PO}_{4}$ $50 \mathrm{mM}, \mathrm{NaCl} 300 \mathrm{mM}$, glycerol 10\%, amd imidazole $10 \mathrm{mM}$. The washing buffer (pH 8.0) comprised $\mathrm{NaH}_{2} \mathrm{PO}_{4} 50 \mathrm{mM}, \mathrm{NaCl} 300 \mathrm{mM}$, glycerol $10 \%$, and imidazole $20 \mathrm{mM}$. Elution buffer ( $\mathrm{pH}$ 8.0) was composed of $\mathrm{NaH}_{2} \mathrm{PO}_{4} 50 \mathrm{mM}, \mathrm{NaCl} 300 \mathrm{mM}$, glycerol $10 \%$, and imidazole $250 \mathrm{mM}$. Desalting buffer ( $\mathrm{pH} 7.4)$ was a mixture of $\mathrm{NaH}_{2} \mathrm{PO}_{4}$, EDTA $1 \mathrm{mM}$, and glycerol $10 \%$.

\section{Cloning and expression of enzymes}

The ole $T_{J E}$ gene was amplified using the previously constructed plasmid pET-28(b)-ole $T_{J E}$ as template and the primer pair as follows: BamHI-ole $T_{J E}$, CGCGGATCCG ATGGCAACACTTAAGAGGGATAAGGGCTTA (the

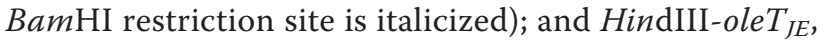
CAATGAAGCTTTTATGTTCTGTCTAC AACTTCGC (the italicized nucleotides denote the HindIII cutting site). For $t l l$ gene cloning, the synthetic $t l l$ gene (Genbank accession number AF054513.1) was used as template for
PCR amplification with primers including BamHI-tll, AGCCAGGATCCGAGTCCTATTCGTCGAGAGGTCT CG and HindIII- $t l l$, GCCGCAA GCTTCTAAAGACAT GTCCCAATTAACCC. These amplified ole $T_{J E}$ and $t l l$ fragments were double digested with BamHI/HindIII and ligated into pACYCDuet-1 and pRSFDuet-1 to create the recombinant plasmids pACYCDuet-ole $T_{J E}$ and pRSFDuet- $t l l$, respectively. The recombinant plasmids were first transformed into competent cells of $E$. coli DH5 $\alpha$ for DNA sequence confirmation, and then transformed into E. coli BL21 (DE3) for cytoplasmic overexpression of $\mathrm{OleT}_{\mathrm{JE}}$ and Tll. For construction of engineered E. coli strains with periplasmic overexpression of lipase, the plasmid pET-22(b) carrying an $\mathrm{N}$-terminal pelB signal sequence was used. The tll fragment from BamHI/HindIII double digested pRSFDuet-tll was inserted into corresponding sites of pET-22(b), resulting in the recombinant plasmid pET-22(b)-tll. The engineered E. coli BL21 (DE3) strains harboring corresponding recombinant plasmids were similarly constructed as described above.

A single colony of the E. coli BL21 (DE3) strain carrying pACYCDuet-ole $T_{J E}$, pRSFDuet-tll, or pET-22(b) based counterparts were used to inoculate the seed culture in $\mathrm{LB}$, and this culture was grown overnight at $37^{\circ} \mathrm{C}$, $200 \mathrm{rpm}$. A $1 \%$ volume of pre-culture was inoculated into 11 of TB medium with appropriate antibiotics (plus $0.5 \mathrm{mM} 5$-aminolevulinic acid and $1 \mathrm{mM}$ thiamine for $\mathrm{OleT}_{\mathrm{JE}}$ expression). When $\mathrm{OD}_{600}$ reached approximately 0.8 , IPTG was added to the final concentration of $0.2 \mathrm{mM}$ for induction of enzyme expression at $18^{\circ} \mathrm{C}$ for $20 \mathrm{~h}$.

\section{Purification of enzymes}

The harvested post-induced cells by centrifugation were resuspended with $40 \mathrm{ml}$ lysis buffer and disrupted using ultra-sonication. Cell slurry was separated into cell-free lysate and cell debris at $10,000 \times g$ for $30 \mathrm{~min}$ at $4^{\circ} \mathrm{C}$. 
The resulted cell-free lysate was gently mixed with $1 \mathrm{ml}$ $50 \% \mathrm{Ni}$-NTA slurry at $4^{\circ} \mathrm{C}$ for $1 \mathrm{~h}$. The mixture was loaded onto a column and washed with 100 to $200 \mathrm{ml}$ washing buffer until no protein eluted in the flowthrough. The $6 \times$ His tagged proteins bound to Ni-NTA resin were eluted with $5 \mathrm{ml}$ elution buffer. The eluted protein fraction was concentrated using Millipore ultrafilter with $30-\mathrm{kDa}$ molecular weight cutoff $(2,000 \times g$, $30 \mathrm{~min}, 4^{\circ} \mathrm{C}$ ) and loaded onto a pre-equilibrated PD-10 column for buffer exchange using desalting buffer. All protein fractions were monitored using Bradford Protein Assay kit. The aliquots of collected fractions were flash frozen by liquid nitrogen and stored at $-80^{\circ} \mathrm{C}$ for later use.

\section{Determination of enzyme concentration}

Diluted purified Ole $_{\mathrm{JE}}$ solution was reduced by adding sodium dithionite prior to the initial scan at 400 to $600 \mathrm{~nm}$ to record the baseline spectrum. Then, the P450 enzyme solution was subjected to $\mathrm{CO}$ bubbling for $40 \mathrm{~s}$ before the second scan to get the reduced CO-bound $\mathrm{P} 450$ spectrum, based on which the functional Ole $\mathrm{T}_{\mathrm{JE}}$ was quantified from the absorbance difference between $A_{450}$ and $A_{490}$ using the molar extinction coefficient of $91 \mathrm{mM}^{-1} \mathrm{~cm}^{-1}$ [29]. The lipase activity was measured as described elsewhere [25].

\section{Whole cell catalyst preparation}

The induced culture broth was centrifuged to harvest fresh wet cells, termed as non-treated cells. These fresh wet cells were subjected to freezing at $-80^{\circ} \mathrm{C}$ for $12 \mathrm{~h}$, and then freeze-drying for $24 \mathrm{~h}$ with lyophilizer to obtain freeze-dried cells.

\section{Enzymatic assays and whole cell biotransformation}

For cell-free enzyme biotransformation, the standard $100-\mu$ hydrolysis reaction assay containing $0.5 \mathrm{mM}$ substrate, $2 \mu \mathrm{M}$ purified Tll in sodium phosphate buffer (100 mM, pH 7.4) was carried out in a 1.5-ml Eppendorf tube at $30^{\circ} \mathrm{C}$ for $6 \mathrm{~h}$. The tested substrates included pure TAGs (fatty acyl chain length of C12, C14, and C16) and oils (microalgae oil, soybean oil, and olive oil). For the tandem reactions of hydrolysis followed by decarboxyl-

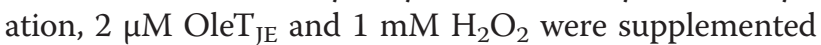
into the hydrolysis reaction while maintaining the total volume of $100 \mu \mathrm{l}$. During optimization of the molar ratio between these two enzymes, the amount of each enzyme was adjusted appropriately.

For resting whole cell biotransformation, various amounts of resting recombinant cells (approximate $50 \mathrm{mg}$ ) with the same activities (in terms of cell-free extract) were added into $500 \mu \mathrm{l}$ sodium phosphate buffer containing $0.5 \mathrm{mM}$ microalgae oil. In the case of lipase-OleT $\mathrm{JE}_{\mathrm{JE}}$ coupled catalysis reaction, $1 \mathrm{mM} \mathrm{H}_{2} \mathrm{O}_{2}$ was supplied. During optimization of the enzyme ratio in the whole cell catalysis, the cell amounts were accordingly adjusted. In the whole cell-mediated recycling biotransformations, E. coli cells were recovered and washed after each batch reaction, and then applied in a new batch reaction. These resting whole cell-mediated biotransformations were performed at $30^{\circ} \mathrm{C}$ for $24 \mathrm{~h}$.

For biotransformation mediated by growing cells in situ, 12-h post-induction cultures were supplemented with $0.5 \mathrm{mM}$ microalgae oil and $1 \mathrm{mM} \mathrm{H}_{2} \mathrm{O}_{2}$ for additional $36-\mathrm{h}$ reaction in shaking flask at $28^{\circ} \mathrm{C}$. All experiments were repeated three times.

\section{Determination of FFA and 1-alkene}

The reactions were quenched and extracted with $100 \mu \mathrm{l}$ ethyl acetate containing $0.5 \mathrm{mM}$ heptadecenoic acid as internal standard for GC-MS analysis. The GC oven equipped with the HP-INNOWAX (Agilent Technologies, Inc., Santa Clara, CA, USA; $30 \mathrm{~m} \times 0.25 \mathrm{~mm} \times 0.25 \mu \mathrm{m}$ ) column was heated using the program as follows: isothermal at $40^{\circ} \mathrm{C}$ for $4 \mathrm{~min}, 40$ to $250^{\circ} \mathrm{C}$ at the rate of $10^{\circ} \mathrm{C} / \mathrm{min}$, and $250^{\circ} \mathrm{C}$ for $15 \mathrm{~min}$. The mass fragment range of 50 to $500 \mathrm{~m} / \mathrm{z}$ was monitored under electron ionization conditions (1741 eV). FFAs and alkenes were quantified using corresponding standard compounds and the internal standard as references.

\section{Additional files}

Additional file 1: Graphical abstract. An artificial two-step biosynthetic pathway for biological production of 1-alkenes using three kinds of natural oils.

Additional file 2: Figure S1. Plasmid construction and purification of TII (A) and OleT JE (B).

Additional file 3: Table S1. Profiles of produced FFAs via lipase TII mediated hydrolysis and produced 1-alkenes via lipase-OleT JE tandem biotransformation. All experiments were performed three times.

\section{Abbreviations}

TAGs: triacylglycerols; TIl: Thermomyces lanuginosus lipase; FFAs: free fatty acids; IPTG: isopropyl $\beta$-D-1-thiogalactopyranoside.

\section{Competing interests}

The authors declare that they have no competing interests.

\section{Authors' contributions}

$J Y$ and SL conceived of the study, designed the experiments, analyzed the data, and wrote the manuscript. JY, YL, CW, and $\mathrm{BH}$ performed the experiments. All authors read and approved the final manuscript.

\section{Acknowledgements}

This work was supported by the Qingdao Institute of Bioenergy and Bioprocess Technology Director Innovation Foundation for Young Scientists (Grant Number: Y37203410U), the Applied Basic Research Programs of Science and Technology of Qingdao (Grant Number: 14-2-4-10-jch), the National Natural Science Foundation of China (Grant Number: NSFC 31270855), and the National High Technology Research and Development Program of China (Grant Number: 2014AA093510). We are also grateful for the funding from 'Recruitment Program of Global Experts, 2012.' We thank Professor Tianzhong Liu at the Qingdao Institute of Bioenergy and Bioprocess Technology, Chinese Academy of Sciences, for providing us with the microalgae oils. 


\section{Author details}

${ }^{1}$ Key Laboratory of Biofuels, and Shandong Provincial Key Laboratory of Energy Genetics, Qingdao Institute of Bioenergy and Bioprocess Technology, Chinese Academy of Sciences, No. 189 Songling Road, 266101 Qingdao, Shandong, China. ${ }^{2}$ University of Chinese Academy of Sciences, No. 19A Yuquan Road, 100049 Beijing, China. ${ }^{3}$ Key Laboratory for Marine Drugs, Department of Pharmacy, Renji Hospital, Shanghai Jiao Tong University School of Medicine, No. 145 Shandongzhong Road, 200127 Shanghai, China.

Received: 16 September 2014 Accepted: 4 February 2015

Published online: 26 February 2015

\section{References}

1. Keasling JD, Chou H. Metabolic engineering delivers next-generation biofuels. Nat Biotechnol. 2008;26:298-9.

2. Atsumi S, Liao JC. Metabolic engineering for advanced biofuels production from Escherichia coli. Curr Opin Biotechnol. 2008;19:414-9.

3. Lee SK, Chou H, Ham TS, Lee TS, Keasling JD. Metabolic engineering of microorganisms for biofuels production: from bugs to synthetic biology to fuels. Curr Opin Biotechnol. 2008;19:556-63.

4. Serrano-Ruiz JC, Ramos-Fernández EV, Sepúlveda-Escribano A. From biodiesel and bioethanol to liquid hydrocarbon fuels: new hydrotreating and advanced microbial technologies. Energy Environ Sci. 2012;5:5638-52.

5. Schirmer A, Rude MA, Li X, Popova E, del Cardayre SB. Microbial biosynthesis of alkanes. Science. 2010;329:559-62.

6. Akhtar MK, Turner NJ, Jones PR. Carboxylic acid reductase is a versatile enzyme for the conversion of fatty acids into fuels and chemical commodities. Proc Natl Acad Sci U S A. 2013;110:87-92.

7. Thomas PH, Sabine M, Karen M, Christoph E, Dagmara MK, George NT, et al. Synthesis of customized petroleum-replica fuel molecules by targeted modification of free fatty acid pools in Escherichia coli. Proc Natl Acad Sci U S A. 2013;110:7636-41.

8. Beller HR, Goh EB, Keasling JD. Genes involved in long-chain alkene biosynthesis in Micrococcus luteus. Appl Environ Microbiol. 2010;76:1212-23.

9. Sukovich DJ, Seffernick JL, Richman JE, Gralnick JA, Wackett LP. Widespread head-to-head hydrocarbon biosynthesis in bacteria and role of OleA. Appl Environ Microbiol. 2010;76:3850-62.

10. Liu Y, Wang C, Yan JY, Zhang W, Guan WN, Lu XF, et al. Hydrogen peroxide-independent production of $a$-alkenes by OleT JE P450 fatty acid decarboxylase. Biotechnol Biofuels. 2014;7:28-39.

11. Kung $Y$, Runguphan W, Keasling JD. From fields to fuels: recent advances in the microbial production of biofuels. ACS Synth Biol. 2012;1:498-513.

12. Peralta-Yahya PP, Zhang FZ, del Cardayre SB, Keasling JD. Microbial engineering for the production of advanced biofuels. Nature. 2012:488:320-8.

13. Steen EJ, Kang Y, Bokinsky G, Hu Z, Schirmer A, McClure A, et al. Microbial production of fatty-acid-derived fuels and chemicals from plant biomass. Nature. 2010;463:559-62.

14. Liu TG, Vora H, Khosla C. Quantitative analysis and engineering of fatty acid biosynthesis in E. coli. Metab Eng. 2010;12:378-86.

15. Lu XF, Vora H, Khosla C. Overproduction of free fatty acids in E. coli: implications for biodiesel production. Metab Eng. 2008;10:333-9.

16. Lennen RM, Pfleger BF. Engineering Escherichia coli to synthesize free fatty acids. Trends Biotechnol. 2012;30:659-67.

17. Lennen RM, Max AK, Kritika K, Robert AZ, Kristin EB, Mary SL, et al. Membrane stresses induced by overproduction of free fatty acids in Escherichia coli. Appl Environ Microbiol. 2011;77:8114-28.

18. Fernandez-Lafuente R. Lipase from Thermomyces lanuginosus: uses and prospects as an industrial biocatalyst. J Mol Catal B Enzym. 2010;62:197-212.

19. Rude MA, Baron TS, Brubaker S, Alibhai M, del Cardayre SB, Schirmer A. Terminal olefin (1-alkene) biosynthesis by a novel P450 fatty acid decarboxylase from Jeotgalicoccus species. Appl Environ Microbiol. 2011;77:1718-27.

20. Yan JY, Yan YJ, Liu SX, Hu J, Wang GL. Preparation of cross-linked lipase-coated micro-crystals for biodiesel production from waste cooking oil. Bioresour Technol. 2011;102:4755-8.

21. Yan JY, Li AT, Xu Y, Ngo Thao PN, Phua SC, Li Z. Efficient production of biodiesel from waste grease: one-pot esterification and transesterification with tandem lipases. Bioresour Technol. 2012;123:332-7.

22. Yan JY, Zheng XL, Li SY. A novel and robust recombinant Pichia pastoris yeast whole cell biocatalyst with intracellular overexpression of a Thermomyces lanuginosus lipase: preparation, characterization and application in biodiesel production. Bioresour Technol. 2014;151:43-8.
23. Luisa G, Ana CO. Microalgae as a raw material for biofuels production. J Ind Microbiol Biotechnol. 2009;36:269-74.

24. Brundiek HB, Evitt AS, Kourist R, Bornscheuer UT. Creation of a lipase highly selective for trans fatty acids by protein engineering. Angew Chem Int Ed. 2012;51:412-4

25. Yan JY, Zheng XL, Du L, Li SY. Integrated lipase production and in situ biodiesel synthesis in a recombinant Pichia pastoris yeast: an efficient dual biocatalytic system composed of cell free enzymes and whole cell catalysts. Biotechnol Biofuels. 2014;7:55-62.

26. Belcher J, McLean KJ, Matthews S, Woodward LS, Fisher K, Rigby SE, et al. Structure and biochemical properties of the alkene producing cytochrome P450 OleT JE (CYP152L1) from the Jeotgalicoccus sp. 8456 bacterium. J Biol Chem. 2014:289:6535-50.

27. You C, Zhang YHP. Self-assembly of synthetic metabolons through synthetic protein scaffolds: one-step purification, co-immobilization, and substrate channeling. ACS Synth Biol. 2013;2:102-10.

28. Zhang YHP, Sun JB, Zhong JJ. Biofuel production by in vitro synthetic enzymatic pathway biotransformation. Curr Opin Biotechnol. 2010;21:663-9.

29. Johnston WA, Huang WL, De Voss JJ, Hayes MA, Gillam EMJ. Quantitative whole-cell cytochrome P450 measurement suitable for high-throughput application. J Biomol Screen. 2008;13:135-41.

\section{Submit your next manuscript to BioMed Central and take full advantage of:}

- Convenient online submission

- Thorough peer review

- No space constraints or color figure charges

- Immediate publication on acceptance

- Inclusion in PubMed, CAS, Scopus and Google Scholar

- Research which is freely available for redistribution 